\title{
CD14/16 Monocyte Profiling in Juvenile Myelomonocytic Leukemia
}

\author{
Manisha Gadgeel ${ }^{1}$, Shruti Bagla ${ }^{2}$, Steven Buck ${ }^{3}$, Mark Shamoun ${ }^{3}$, and Yaddanapudi \\ Ravindranath ${ }^{1}$ \\ ${ }^{1}$ Wayne State University School of Medicine \\ ${ }^{2}$ Wayne State University \\ ${ }^{3}$ Children's Hospital of Michigan
}

May 6, 2020

\begin{abstract}
Monocyte subset analysis by flow cytometry has been shown to be a useful diagnostic tool in chronic myelomonocytic leukemia in adults. An increase in the classical monocyte fraction (CD14++/CD16-) greater than $94.0 \%$ of total monocytes is considered highly sensitive and specific in distinguishing CMML from other myeloproliferative disorders. In a pilot study of juvenile myelomonocytic leukemia cases, we noted that CD14++/CD16- monocyte fraction was $>95 \%$ in de novo JMML with somatic PTPN11 mutations but normal in those with monosomy 7 or Noonan syndrome. Monocyte subgroup profiling by itself is not diagnostic of JMML but may distinguish molecular subgroups within JMML.
\end{abstract}

\section{Introduction}

Abnormal monocyte proliferation is a central feature of both juvenile myelomonocytic leukemia (JMML) in children and chronic myelomonocytic leukemia (CMML) in adults. Flow cytometry based monocyte profiling has been found to be a useful aid in the diagnosis of CMML. ${ }^{1-5}$ Monocytes can be classified into three functionally and immunologically distinct cell populations based on expression of CD14 and CD16 surface markers: classical monocytes (CD14++/CD16-), intermediate monocytes (CD14+/CD16++), and non-classical monocytes (CD14-/CD16++) ${ }^{6-9}$ Classical monocytes comprise about $90 \%$ of the circulating monocytes with only $10 \%$ intermediate and non-classical monocytes. ${ }^{7},{ }^{10}$ Absolute count of monocyte subsets by flow cytometry can vary in various disease conditions and provide clinically useful information in management of diseases. ${ }^{7}, 11,12$ An increase in the classical monocyte fraction (CD14++/CD16-) greater than $94.0 \%$ of total monocytes is considered highly sensitive and specific in distinguishing CMML from other myeloproliferative disorders. ${ }^{2,3,9} \mathrm{~A}$ specificity of $97 \%$ in peripheral blood (PB) and $100 \%$ in bone marrow (BM) samples was observed in one study. ${ }^{4}$

While the distribution and expression of CD14/CD16 surface markers is very well characterized in adult CMML, no such categorization has been reported in JMML. In this pilot study, we evaluated the monocyte partitioning in 14 JMML samples (PB and BM).

\section{Methods}

\section{Samples}

Between the years 1979-2019, total of 14 patients were clinically diagnosed with JMML at Children's Hospital of Michigan, Detroit MI. Frozen archived mononuclear cells isolated from PB or BM samples were available for 12 patients and fresh samples were available for the 2 patients presented in 2019. The study was approved by the Institutional Review Board at Wayne State University.

\section{Monocyte profiling}


CD14/CD16 monocyte profiling was carried out by flow cytometry. Briefly, cryopreserved mononuclear cells were rapidly thawed under warm water, washed with complete medium and resuspended in staining buffer. For fresh samples, mononuclear cells from heparinized peripheral blood or bone marrow samples were obtained by density gradient separation using Fico/Lite-LymphoH (Atlanta Biologicals; Flowery Branch, GA), washed in complete medium (RPMI1640 + 10\% fetal bovine serum + gentamycin) and re-suspended in phosphate-buffered saline (PBS) $+30 \%$ adult bovine serum as a blocking agent for non-specific staining of immunoglobulins. Cell viability was assessed by trypan blue staining. Surface markers were assessed for 3 -color immunophenotyping by incubating mononuclear cells for 20 minutes in the dark with fluorescenceconjugated monoclonal antibodies against CD45 (PC5), CD16 (FITC) and CD14 (PE) (Beckman Coulter; Brea, CA), followed by washing in PBS and re-suspension in PBS $+0.4 \%$ formaldehyde as a fixative prior to acquisition using a Coulter XL-MCL flow cytometer (Beckman Coulter; Brea, CA) equipped with an Argon laser. Monocyte populations were gated based on CD45/SS and CD14/SS analysis. Thereafter, CD14/CD16 double parameter plots were used to further define the three subsets of mature monocytes, CD14++/CD16- classical (MO1), CD14+/CD16++ intermediate (MO2), and CD14-/CD16++ non-classical (MO3) monocytes. ${ }^{6-9}$ The distribution of monocytes was reported as percentages within the total monocyte gate.

\section{Results and Discussion:}

Samples from 14 cases diagnosed to have JMML between 1979 and December 2019 were available for testing; all patients were under age 13; 3 of these cases were included in previous publications. ${ }^{13,14}$ Four of the 12 archived samples showed increased necrosis on flow cytometry and therefore were excluded from the analysis. Figure 1 shows CD14/CD16 monocyte profiling on the remaining 10 JMML samples (8 archival samples; 2 fresh samples) along with the associated JMML mutation.

In four patients (Panel A), MO1 monocytes (CD14++/CD16) were $>95 \%$ of total monocytes (range 95.1 to $98.8 \%$ ). Three of these had somatic PTPN11 mutations (PTPN11 p.A72V,PTPN11 p.E76A, PTPN11 p.A72T), while one case had a novel $C B L$ p.L62F mutation. No such increase in MO1 population was observed in any of the four Monosomy-7 patients (despite having concurrent PTPN11 or KRAS mutations) or the two Noonan syndrome cases with PTPN11 p.E73I mutation (Panel B). This distinct clustering of JMML cases based on monocyte subsets was unexpected. Molecular studies have identified clinically significant clustering of JMML cases with somatic PTPN11 mutations associated with poorer prognosis than those with germline mutations as seen in Noonan syndrome. Epigenetic studies have also reported increased global 5-methylcytosine in JMML associated with somatic PTPN11 mutations as compared to the Noonan syndrome cases. ${ }^{15,16}$

In summary, this pilot study is the first to evaluate monocyte subsets in JMML and correlate with underlying JMML genetics. Flow-based monocyte profiling of JMML patients is not diagnostic of JMML per se but the presence of $>95 \%$ classical monocytes identifies a subset of cases with underlying deleterious somatic PTPN11 mutations. In adult CMML, the monocyte subset repartitioning showed normalization in patients who responded to hypomethylating agents (HMA). ${ }^{3}$ Thus, monocyte profiling may be a useful tool in characterizing the abnormal monocyte proliferation in JMML and for therapeutic monitoring.

Conflict of interest:The authors have no conflicts of interest

\section{Acknowledgments:}

This study was funded by a grant from The Children's Hospital of Michigan Foundation and funds from the Georgie Ginopolis Chair in Pediatric Cancer and Hematology award to Dr. Y Ravindranath.

\section{References}

1. Xu Y, McKenna RW, Karandikar NJ, Pildain AJ, Kroft SH. Flow cytometric analysis of monocytes as a tool for distinguishing chronic myelomonocytic leukemia from reactive monocytosis. Am J Clin Pathol . Nov 2005;124(5):799-806. doi:10.1309/HRJ1-XKTD-77J1-UTFM 
2. Talati C, Zhang L, Shaheen G, et al. Monocyte subset analysis accurately distinguishes CMML from MDS and is associated with a favorable MDS prognosis. Blood. Mar 30 2017;129(13):1881-1883. doi:10.1182/blood-2016-12-753210

3. Selimoglu-Buet D, Wagner-Ballon O, Saada V, et al. Characteristic repartition of monocyte subsets as a diagnostic signature of chronic myelomonocytic leukemia. Blood . Jun 4 2015;125(23):3618-26. doi:10.1182/blood-2015-01-620781

4. Picot T, Aanei CM, Flandrin Gresta P, et al. Evaluation by Flow Cytometry of Mature Monocyte Subpopulations for the Diagnosis and Follow-Up of Chronic Myelomonocytic Leukemia. Front Oncol . 2018;8:109. doi:10.3389/fonc.2018.00109

5. Hudson CA, Burack WR, Leary PC, Bennett JM. Clinical Utility of Classical and Nonclassical Monocyte Percentage in the Diagnosis of Chronic Myelomonocytic Leukemia. Am J Clin Pathol . Aug 30 2018;150(4):293-302. doi:10.1093/ajcp/aqy054

6. Passlick B, Flieger D, Ziegler-Heitbrock HW. Identification and characterization of a novel monocyte subpopulation in human peripheral blood. Blood. Nov 15 1989;74(7):2527-34.

7. Ziegler-Heitbrock L, Ancuta P, Crowe S, et al. Nomenclature of monocytes and dendritic cells in blood. Blood. Oct 21 2010;116(16):e74-80. doi:10.1182/blood-2010-02-258558

8. Grage-Griebenow E, Zawatzky R, Kahlert H, Brade L, Flad H, Ernst M. Identification of a novel dendritic cell-like subset of CD64(+) / CD16(+) blood monocytes. Eur J Immunol. Jan 2001;31(1):48-56. doi:10.1002/1521-4141(200101)31:1\&\#60;48::aid-immu48\&\#62;3.0.co;2-5

9. Patnaik MM, Timm MM, Vallapureddy R, et al. Flow cytometry based monocyte subset analysis accurately distinguishes chronic myelomonocytic leukemia from myeloproliferative neoplasms with associated monocytosis.Blood Cancer J . Jul 21 2017;7(7):e584. doi:10.1038/bcj.2017.66

10. Wong KL, Tai JJ, Wong WC, et al. Gene expression profiling reveals the defining features of the classical, intermediate, and nonclassical human monocyte subsets. Blood . Aug 4 2011;118(5):e16-31. doi:10.1182/blood-2010-12-326355

11. Ozanska A, Szymczak D, Rybka J. Pattern of human monocyte subpopulations in health and disease. Scand J Immunol . Apr 3 2020;doi:10.1111/sji.12883

12. Carstensen M, Christensen T, Stilund M, Moller HJ, Petersen EL, Petersen T. Activated monocytes and markers of inflammation in newly diagnosed multiple sclerosis. Immunol Cell Biol . Apr 6 2020;doi:10.1111/imcb.12337

13. Inoue S, Shibata T, Ravindranath Y, Gohle N. Clonal origin of erythroid cells in juvenile chronic myelogenous leukemia. Blood. Mar 1987;69(3):975-6.

14. Inoue S, Ravindranath Y, Thompson RI, Zuelzer WW, Ottenbreit MJ. Cytogenetics of juvenile type chronic granulocytic leukemia.Cancer . May 1977;39(5):2017-24. doi:10.1002/10970142(197705)39:5<2017::aid-cncr2820390518>3.0.co;2-z

15. Niemeyer CM, Flotho C. Juvenile myelomonocytic leukemia: who's the driver at the wheel? Blood . Mar 7 2019;133(10):1060-1070. doi:10.1182/blood-2018-11-844688

16. Murakami N, Okuno Y, Yoshida K, et al. Integrated molecular profiling of juvenile myelomonocytic leukemia. Blood. Apr 5 2018;131(14):1576-1586. doi:10.1182/blood-2017-07-798157

Figure Legend:

Figure 1: CD14/16 monocyte profiling in JMML (A) 4 cases of JMML with increased classical monocytes, $>95 \%$, (B) JMML cases with normal monocyte profile. * indicates BM sample 


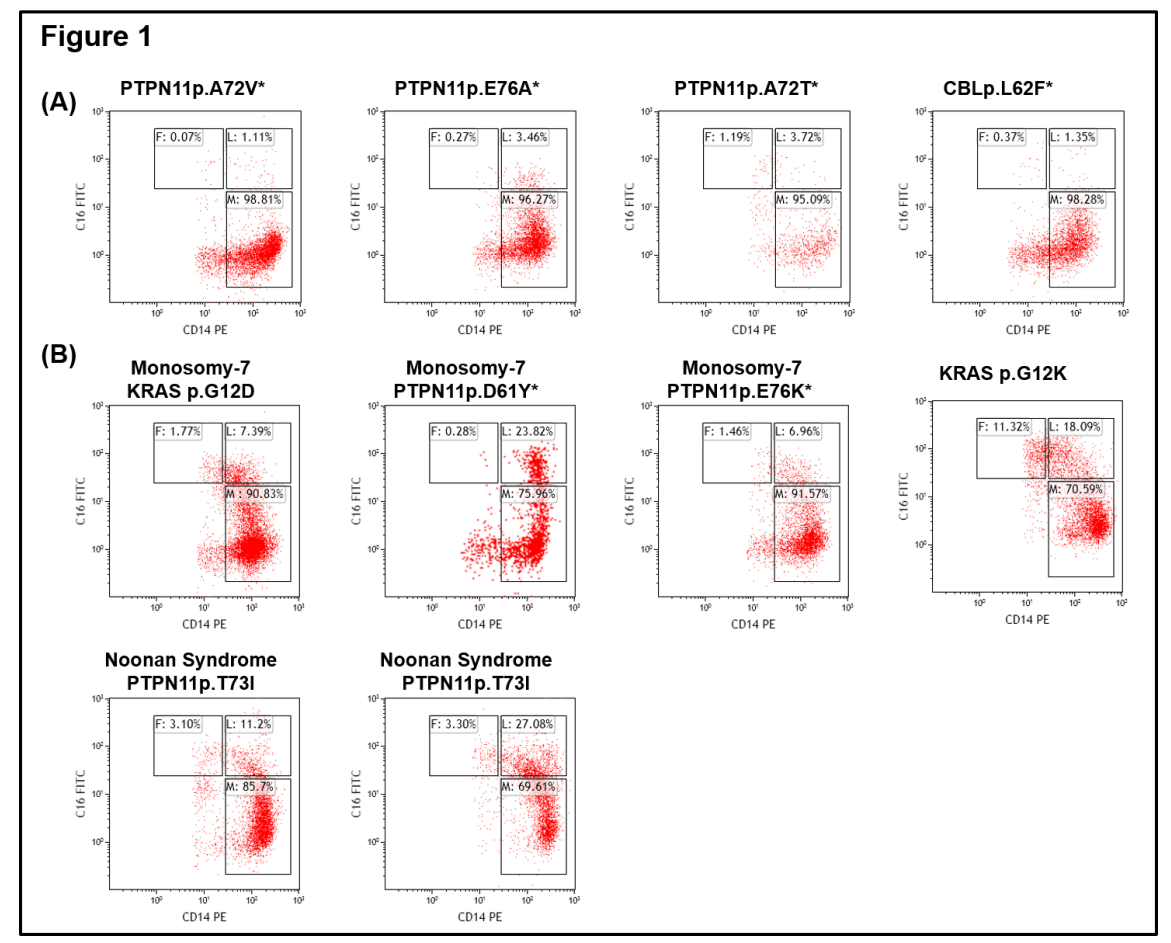

